\title{
STUDY OF A FAR INFRARED CAVITY AT THE GALACTIC PLANE
}

\author{
A. K. Gautam* and B. Aryal** \\ *Bhaktapur Multiple Campus, Bhaktapur. \\ ** Central Department of Physics, T.U., Kirtipur.
}

\begin{abstract}
A far infrared dust cavity nearby AGB star namely AGB19+26 found to be located, centered at R.A.(J2000)= $20^{\mathrm{h}} 02^{\mathrm{m}} 02.8^{\mathrm{s}}$ and Dec. $(\mathrm{J} 2000)=26^{0} 52^{\prime} 36.8^{\prime \prime}$.Distribution of Planck function, dust color temperature, dust mass and visual extinction of the far infrared cavity was studied. This cavity was found to have following properties: (a) it is located nearby AGB star named AGB19+26around far infrared loopG064-01 at 2000 pc, (b) it is close to the Galactic plane at galactic latitude $-1.2^{0}$ therefore the radiation field is strong and (c) the diameter of the cavity is greater than $0.2^{0}$ on IRAS. The dust color temperatures are found to be in the range $21.48 \pm 0.25 \mathrm{~K}$ to $22.03 \pm 0.12 \mathrm{~K}$. Planck function was found to be non-uniform along its extension and compression, suggesting that the dust and grains are not in the local thermodynamic equilibrium, possibly because of external cause. It is therefore, a deviation from the Gaussian distribution is noted in the histogram of dust color temperature. It is concluded that the cavity was formed due to the high pressure events occurred in the past.
\end{abstract}

Keywords: Dust color temperature; Far infrared cavity; Dust mass; Planck function

\section{INTRODUCTION}

Asymptotic giant branch (AGB) stars are low and intermediate mass stars driven by nuclear burning. They are the final nuclear burning evolution stage of low and intermediate mass stars and is characterized by nuclear burning of hydrogen and helium in thin shells on top of electron-degenerate core of carbon and oxygen. This stage is characterized by low surface effective temperatures ( $2000 \mathrm{~K}$ to $3500 \mathrm{~K})$, high luminosity $(\sim 1000 \mathrm{~L} \odot$ to $10,000 \mathrm{~L} \odot$ ), intense mass loss (from $10^{-7}$ to $10^{-4} \mathrm{M} \odot \mathrm{yr}^{-1}$ ) and a slow dust-driven wind ${ }^{1}$. AGB stars are considered the primary source of dust in the interstellar medium $(\text { ISM })^{2,3}$.

Most AGB stars are identified as long-period variables (LPVs) with large amplitude pulsation. The strong pulsation produces shock waves which extend the outer layer of the AGB star for better condition of dust formation ${ }^{4}$. The main site of dust formation is believed to be the cool envelopes around AGB stars and the envelopes are chemically fresh because of the strong binding force of $\mathrm{CO}$ molecules. The radiation pressure produced on newly formed dust grain may drive dusty stellar winds with high mass loss rates of $\left(10^{-6} \text { to } 10^{-4} \mathrm{M} \odot \mathrm{yr}^{-1}\right)^{5}$. Due to such dusty stellar winds and evolution of the central during AGB phase, cool and slowly expanding $(10 \mathrm{~km} / \mathrm{s}$ to $30 \mathrm{~km} / \mathrm{s})$ dust envelope are formed around the AGB stars. Furthermore, AGB stars are characterized by dynamical processes such as pulsation, shockwave, dust formation and mass loss rate. The spectral features are significantly different for stars with Oxygen-rich and Carbon-rich atmospheres.

During the evolution of $\mathrm{AGB}$, the stars also evolve chemically, starting with oxygen-rich atmospheres and Helium burning forms ${ }^{12} \mathrm{C}$, which is dredged up to the stellar surface by strong convection currents in the mantle so that carbon is injected into the stellar atmosphere. The stability of the $\mathrm{CO}$ molecule in the stellar atmosphere that

Author for Correspondence: A.K. Gautam, Department of Physics, Bhaktapur M. Campus, Bhaktapur, T.U., Nepal. Email: arjungautamnjj@gmail.com

Received: 14 Aug 2020; First Review: 29 Aug 2020; Second Review: 19 Sep 2020; Accepted: 22 Sep 2020

Doi: https://doi.org/10.3126/sw.v14i14.34990 
is the carbon -oxygen ratio $(\mathrm{C} / \mathrm{O})$ controls the chemistry around the star. Therefore AGB stars can either be oxygen-rich or carbon-rich depending on their ratio. For the O-rich AGB stars $\mathrm{C} / \mathrm{O}$ can vary from approximately $(\mathrm{C} / \mathrm{O} \approx 0.4)$ to just less than unity, for C-rich stars, the ratio $\mathrm{C} / \mathrm{O}$ is greater than unity. Other nuclear processes (e.g. the s-process) also occur in the He- and H-burning shells of AGB stars where other new elements are also dredged-up and enrich the dust formation region. ${ }^{3}$

\section{Far Infrared Cavities and Far Infrared Loops}

During stellar evolution, stars are changed from one stage to different stages. Out of them, AGB star is the final nuclear burning stage which emits wind. It means AGB stars are main source of dust in the interstellar medium. That wind emitted from high pressure driven events say AGB stars or pulsars or white dwarf or planetary nebula sweep dust as a result cavities are formed. Such cavities stir the ISM which regulates star formation rate. It means cavities are also one important part of stellar evolution. Far infrared cavities are the dusty environment in the far infrared region in the interstellar medium. They provide so many information about new processing stars and previously formed stars. If the cavities are stable then we can say that forming stars and formed stars have longer life. If their distribution of mass follow cosmological

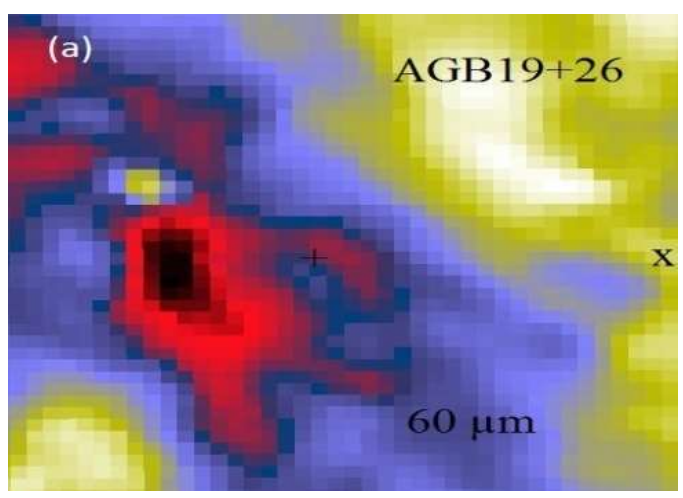

principle, they will be stable and have longer life and so on. It is suitable to observe dust particles which absorbs ultraviolet radiation and emits infrared radiation.

In all-sky survey ${ }^{6},{ }^{7}$ identified 462 FIR loops. Loops are those region in the interstellar medium where the value of flux density at particular wavelength is at least two times less than that of its surroundings. They found 317 loops in the 1st,3rd, and 4th Galactic quadrants, and the 145 loops in 2nd Galactic quadrant. They also studied far-infrared (FIR) properties of the loops and concluded that these structures are formed by high pressure, supernovae explosion, stellar wind, etc. A sort of gas and dust structure such as cavities, filament, loops and shells; applied to as the "cosmic Bubble Bath"can form in the cold Interstellar Medium (ISM).

In the present work, we studied dust color temperature, dust mass, visual extinction and Planck function distributions. Plank function distribution along major and minor diameters are special focus.

\section{Methods}

A far infrared cavity-like structure around AGB star has found in both 60 and $100 \mu \mathrm{m}$ IRAS maps. A method for calculation of dust color temperature, dust mass and visual extinction of the dusty environment around Asymptotic Giant Branch named AGB19+26 has described briefly.

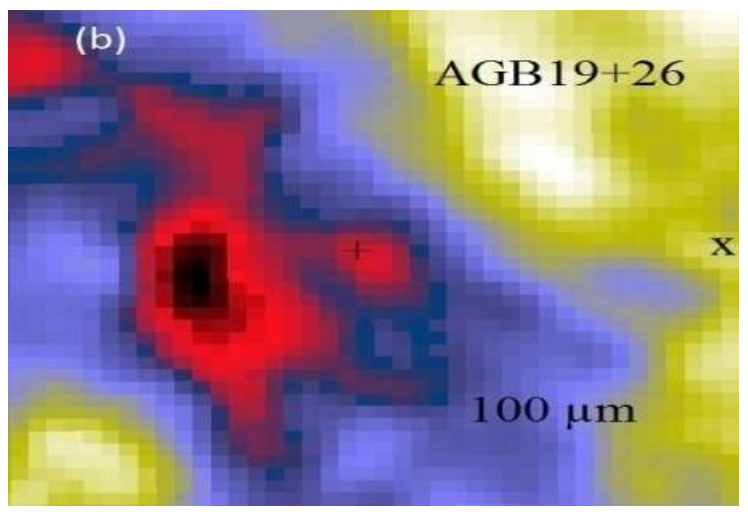

Figure 1: (a) and (b) are the JPEG images of the far infrared cavity at60 $\mu m$ and $100 \mu \mathrm{m}$ IRAS maps around the AGB $19+26$ centered at R.A. $(J 2000)=20^{h} 02^{m} 02.8^{s}$ and Dec. $(J 2000)=26^{0} 52^{\prime} 36.8^{\prime \prime}$.

\section{Dust Color Temperature and Planck Function}

The dust color temperature of the FIR cavity was calculated using 60 and $100 \mu \mathrm{m}$ IRIS flux densities ${ }^{8}$. For it, the method proposed by them was followed and later it was improved. 9,10 They derived an expression for dust color temperature $\mathrm{T}_{\mathrm{d}}$ as 
$\mathrm{T}_{\mathrm{d}}=\frac{-96}{\ln \left\{\mathrm{R} \times 0.6^{(3+\beta)}\right\}}$, where, $\mathrm{R}=\frac{\mathrm{F}(60 \mu \mathrm{m})}{\mathrm{F}(100 \mu \mathrm{m})}$

where $\beta$ is the spectral emissivity index depends on dust grain properties like composition, size, and compactness.

For a pure blackbody, $\beta=0$, the amorphous layer-lattice matter has $\beta \sim 1$, and the metals and crystalline dielectrics have $\beta \sim 2$ which is used in our calculations.

The value of Planck function depends on the wavelength (frequency), and hence temperature. Finally it is used to calculate the dust mass. The Planck function is given by the ${ }^{11,}$

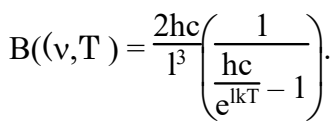

where, $\mathrm{h}=$ Planck constant, $\mathrm{c}=$ velocity of light, $v=$ frequency at which the emission is observed, $\mathrm{T}=$ the average dust color temperature of the region.

\section{Dust Mass and Visual extinction}

For the calculation of dust mass, flux density $\left(\mathrm{F}_{v}\right)$ at 100 $\mu \mathrm{m}$ is needed and we use the expression given by ${ }^{12}$,

$\mathrm{M}_{\text {dust }}=\frac{4 \mathrm{ar}}{3 \mathrm{Q}_{\mathrm{n}}}\left|\frac{\mathrm{S}_{\mathrm{n}} \mathrm{D}^{2}}{\mathrm{~B}(\mathrm{n}, \mathrm{T})}\right|$

where, weighted grain size $(\mathrm{a})=0.1 \mu \mathrm{m}$, grain density $(\rho)$ $=3000 \mathrm{~kg} \mathrm{~m}^{-3}$, grain emissivity $\left(\mathrm{Q}_{v}\right)=0.0010$ (for 100 $\mu \mathrm{m})^{13}$. So the equation (3) reduces to

$\mathrm{M}_{\text {dust }}=0.4\left[\frac{\mathrm{S}_{\mathrm{n}} \mathrm{D}^{2}}{\mathrm{~B}(\mathrm{n}, \mathrm{T})}\right]$

We use equation (4) to calculate dust mass of the cavity.

For estimation of visual extinction, have provided an empirical formula. ${ }^{8}$ According to them, we have

$A_{V}(\operatorname{mag})=15.078\left[1-\exp \left(-\tau_{100} / 641.3\right)\right]$

Where
$\tau_{100}=\frac{F_{\lambda}(100 \mu \mathrm{m})}{\mathrm{B}_{\lambda}(100 \mu \mathrm{m})}$

is optical depth at $100 \mu \mathrm{m}$ wavelength. Here $F_{\lambda}$ is flux density and $\mathrm{B}_{\lambda}$ is Planck function at $100 \mu \mathrm{m}$ wavelength.

\section{Result and Discussion}

\section{Structure}

From the systematic search on IRAS maps, an isolated far infrared cavity in $100 \mu \mathrm{m}$ and $60 \mu \mathrm{m}$ IRAS maps at R.A. $(\mathrm{J} 2000)=20^{\mathrm{h}} 02^{\mathrm{m}} 02.8^{\mathrm{s}}$ and Dec. $(\mathrm{J} 2000)=26^{0} \quad 52^{\prime}$ 36.8" was found which is located around AGB star AGB23+60. Using ALADIN2.5 software, size, dust color temperature, Planck function, dust mass and visual extinction of the cavity have been measured. JPEG images of the cavity at $60 \mu \mathrm{m}$ and $100 \mu \mathrm{m}$ IRAS map are shown in the Fig.1 (a) and (b) respectively. After calculation of major and minor diameter, size of the structure of the cavity was found to be $0.5^{0} \times 0.2^{0}$.

\section{Distribution of Flux Density}

By using ALADIN2.5 software, flux densities at $60 \mu \mathrm{m}$ and $100 \mu \mathrm{m}$ have been measured. Distribution of flux density within the contour of the region of interest was studied. A graph is plotted between $F(100)$ and $F(60)$ which is shown in Fig.2(a). From the linear fit, slope of the line was 0.18 , correlation coefficient $(R)=0.93$. The linear equation of the fitted line is, $F(60)=-1.57+$ $0.18 \mathrm{~F}(100)$. Using the slope of best fitted plot, average dust color temperature is calculated as $22.49 \mathrm{~K}$ which is used to calculate error in calculated dust color temperature.

Again distribution of flux density at $100 \mu \mathrm{m}$ within the contour level with right ascension (R.A.) and declination (Dec.) are plotted and shown in Fig.2(b). Graph shows that all the fluxes from minimum to maximum lie within the contour level. Maximum flux regions lie at the south-east part of the contour. 


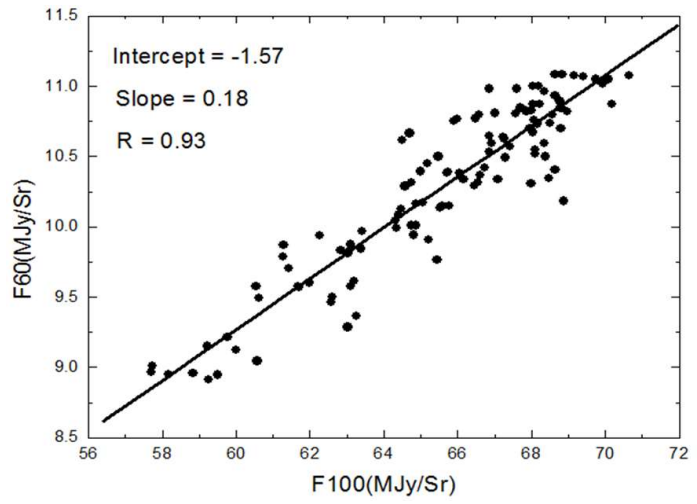

(a)

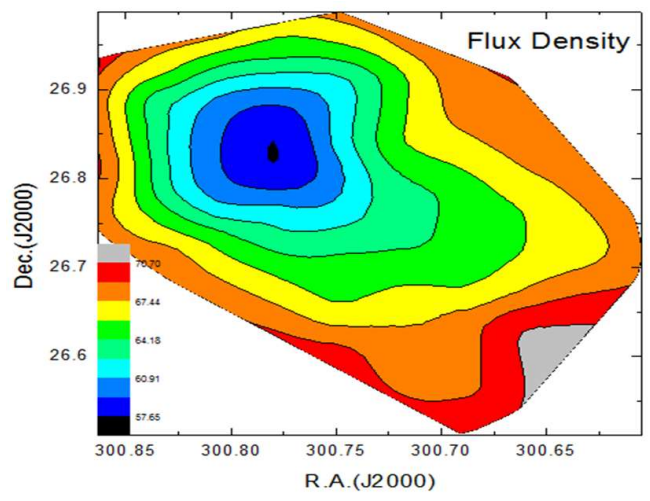

(b)

Figure 2: (a) The $100 \mu \mathrm{m}$ verses $60 \mu \mathrm{m}$ flux density in the region of interest and (b) Contour map at $100 \mu \mathrm{m}$ flux density where the AGB star is located at the center R.A. $(J 2000)=20^{h} 02^{m} 02.8^{s}$ and Dec. $(J 2000)=26^{0} 52^{\prime} 36.8^{\prime \prime}$.

\section{Dust color Temperature and its Variation}

For the calculation of dust color temperature, we used the IRAS $100 \mu \mathrm{m}$ and $60 \mu \mathrm{m}$ FITS images and choose the value of $\beta=2 .{ }^{9}$ Variation of temperature with corresponding R.A.(J2000) and Dec. (J2000) are plotted by using ORIGIN 8.0

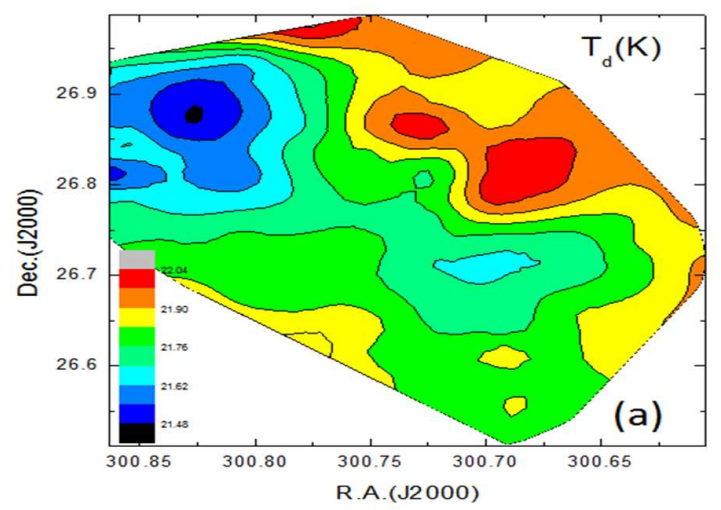

and the graph is shown in Fig. 3 (a). Graph shows that temperature distributions are in separate cluster but minimum temperature region is little bit shifted from minimum flux density which is unusual behaviour. Such type of nature is obtained due to high mass loss by external factors.

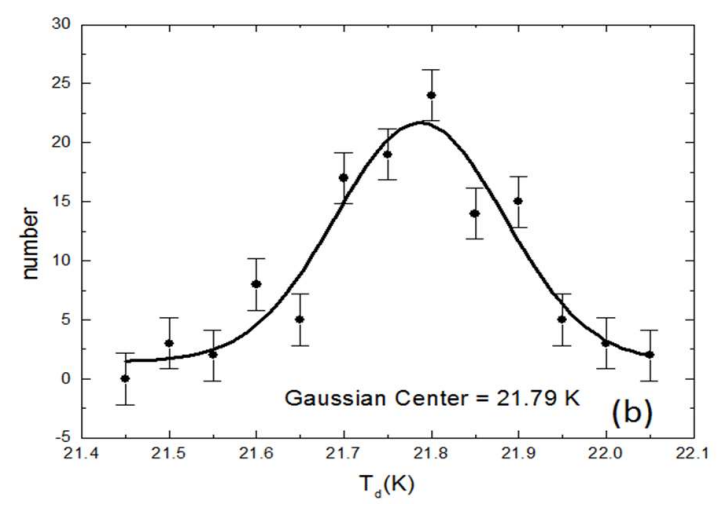

Figure 3: (a) Contour map of dust color temperature and (b) Gaussian fit of dust color temperature. The far infrared cavity is centered at R.A. $(J 2000)=20^{h} 02^{m} 02.8^{s}$ and Dec. $(J 2000)=26^{0} 52^{\prime} 36.8^{\prime \prime}$.

The minimum and maximum dust color temperature was found in the range $21.48 \pm 0.25 \mathrm{~K}$ to $22.03 \pm 0.12 \mathrm{~K}$ respectively with offset value $0.55 \mathrm{~K}$. Such low offset temperature variation shows that there is symmetric outflow or symmetric distribution of density and temperature. It further suggests that particles are independently vibrating. Gaussian fit of dust color temperature is more or less following the Gaussian distribution with positive skewness. When this result is compared with the result obtained in ${ }^{14}$ where temperature variation is $20 \mathrm{~K}$ to $22 \mathrm{~K}$ so our result is also comparable with that result. In the contour map, minimum flux and minimum temperature region are shifted which is due to some external factors possibly due to AGB wind. 


\section{Dust Mass Estimation and its variation}

Since the distance of the structure is $2000 \mathrm{pc}^{7}$ so, by using the temperature of each pixel and corresponding distance of the structure, we calculated mass of each pixel. Total

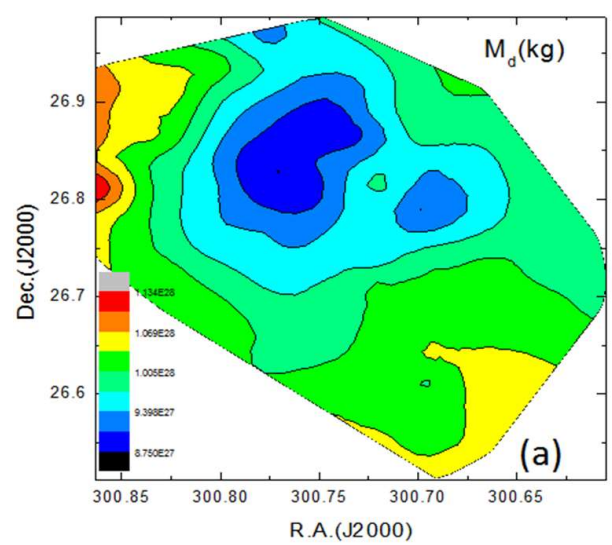

mass of the structure is $1.16 \times 10^{30} \mathrm{~kg}$ i.e $0.58 \mathrm{M} \odot$. But mass of dust obtained around white dwarf WD 1003-44 in ${ }^{14}$ is $0.08 \mathrm{M} \odot$. It means, mass of dust cavity around AGB Star is found to be less than White Dwarf.

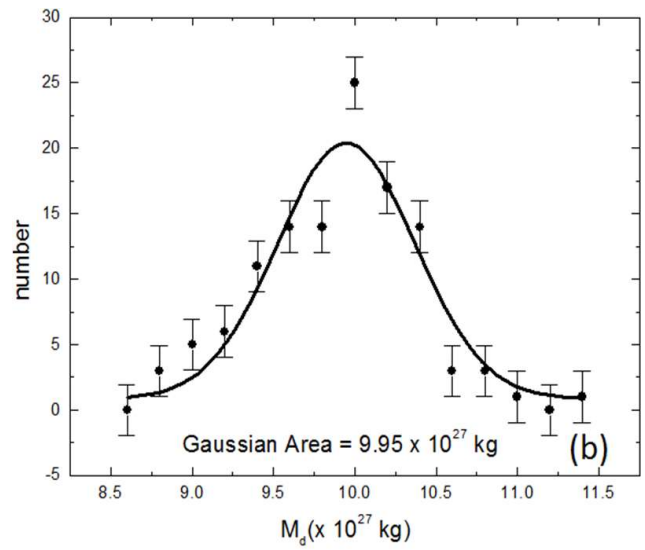

Figure 4: (a) contour map of dust mass and (b) Gaussian fit of dust mass. The far infrared cavity is centered at R.A. $(J 2000)=20^{h} 02^{m} 02.8^{s}$ and Dec. $(J 2000)=26^{0} 52^{\prime} 36.8^{\prime \prime}$.

Distribution of dust mass in the contour map is shown in figure 4(a) which shows different behavior against cosmological principle i.e., minimum temperature region is not denser and vice-versa which is unusual trend. Such behavior is noticed due to effect of high pressure driven events by nearby AGB stars. Figure 4(b) is the Gaussian fit where the data more or less are Gaussian with positive skewness.

\section{Distribution of Planck function with Diameters}

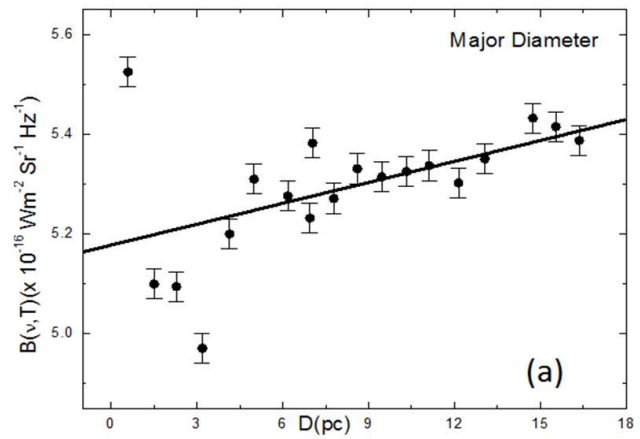

Distribution of Planck function along extension and compression are shown in Fig. 5(a) and (b) where the data are distributed randomly with correlation coefficient $(\mathrm{R})=$ 0.51 in case of major diameter and $\mathrm{R}=0.97$ in case of minor diameter. There is no systematic trend of their distribution in both cases. It means distribution of Plank function is non uniform and is not following the Maxwell velocity distribution which is possibly due to nearby AGB wind.

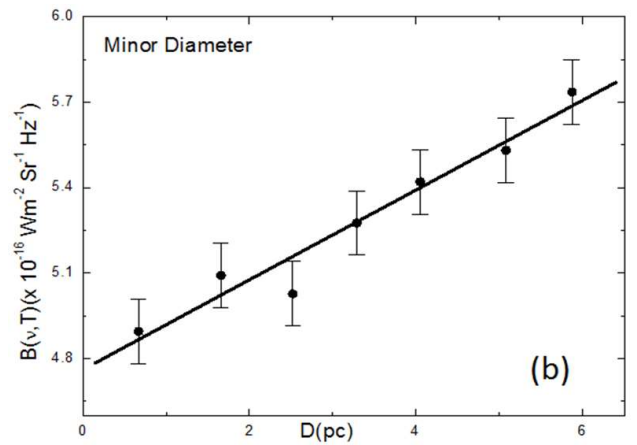

Figure 5: (a) Linear fit of scattered plot between major diameter and Planck function and (b) Linear fit of scattered plot between minor diameter and Planck function of the cavity centered at R.A. $(J 2000)=20^{h} 02^{m} 02.8^{s}$ and Dec. $(J 2000)=26^{0}$ $52^{\prime} 36.8^{\prime \prime}$. 


\section{Variation of Visual Extinction with Dust Color Temperature}

Figure 6(a) is the contour map of visual extinction. Linear fit of the scattered plot between visual extinction and dust color temperature is shown in Fig. 6(b). It shows a systematic trend with moderate correlation coefficient i.e., -0.39 , which shows that there is best correlation between

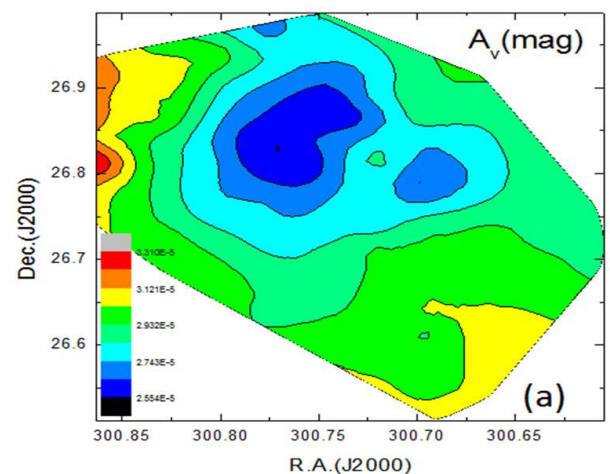

the data. From the linear fit, we found a new relation between visual extinction and dust color temperature. The relation between visual extinction and dust color temperature is $\left(A_{V} \times T_{d}\right)=0.72 \times 10^{-4}$ which also lies in the range proposed by ${ }^{15}$. When it is compared with the contour map of dust color temperature, it is found that higher the dust color temperature, lower the visual extinction and vice-versa.

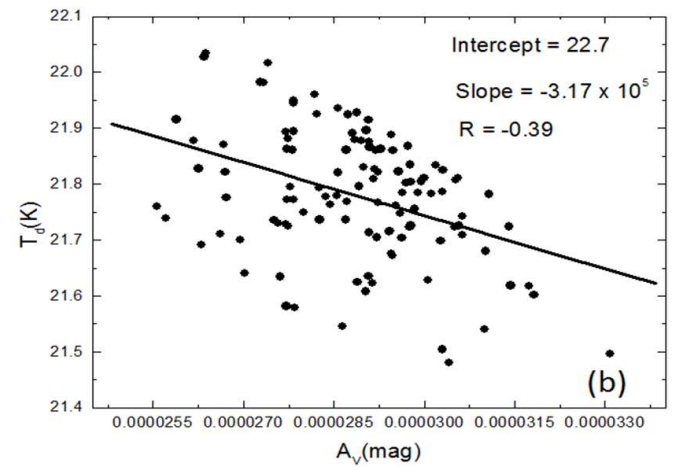

Figure 6: (a) contour map of visual extinction and (b) linear fit of the scattered plot between visual extinction and dust color of the far infrared cavity centered at R.A. $(J 2000)=20^{h} 02^{m} 02.8^{s}$ and Dec. $(J 2000)=26^{0} 52^{\prime} 36.8^{\prime \prime}$.

\section{CONCLUSION}

The physical properties of the far infrared cavity around the AGB19+26 star have been measured and analyzed. Specially, distribution of flux density, dust color temperature, Planck function, dust mass and visual extinction of the cavity have been studied. Results are concluded as follows:

1. Minimum and maximum dust color temperature were found to be $21.48 \pm 0.25 \mathrm{~K}$ and $22.03 \pm 0.12 \mathrm{~K}$ respectively with offset value $0.55 \mathrm{~K}$. Such low off set value suggests that the cavity might be in thermodynamic equilibrium. The dust color maps compliment the dust mass maps in the FIR cavity. The minimum temperature region was found to be massive supporting the cosmological principle.

2. Moderate correlation was noticed between the visual extinction and dust color temperature. The product of dust color temperature and visual extinction was found to be $\left(0.72 \times 10^{-4}\right) \mathrm{K}$ mag which is very less than 1 . Contour map of dust color temperature and visual extinction showed that higher the dust color temperature, lower the visual extinction and vice-versa.
3. A fluctuation in the distribution of Planck function along both diameters of the FIR cavity was noticed, suggesting that the dust particles are oscillating nonuniformly.

4. Total mass of the structure is $1.16 \times 1030 \mathrm{~kg}$ i.e 0.58 $\mathrm{M} \odot$. Distribution of dust mass is supporting cosmological principle. This work will be intended to study the cavities in different bands using IRAS, AKARI and WISE survey in the future.

\section{ACKNOWLEDGEMENTS}

We are grateful to Prof. R. Weinberger, Department of Astro-Particle Physics, Innsbrck University, for invoking us to work on dusty environments around AGB stars. This research has made use of Sky-View Virtual Observatory, Aladin v2.5 and NASA/IPAC Extragalactic Database(NED).

\section{REFERENCE}

1. Habing, H. J. 1996. Circumstellar envelopes and Asymptotic Giant Branch stars. Astronomy and Astrophysics Reviews. 7: 97-207. doi: 10.1007/PL00013287 
2. Habing, H.J., \& Olofsson,H.(Eds.). 2003. Asymptotic giant branch stars.

3. Herwig, F. 2005. Evolution of Asymptotic Giant Branch Stars. Annual Review of Astronomy and Astrophys.43: 435-479. doi: 10.1146/annurev.astro.43.072103.150600

4. Jones,T.W.,Ney,E.P.,\& Stein,W.A. 1981. Pulsations Grain Condensation and Mass Loss in Long-Period Variable Stars. Astrophysical journal. 250: 324. doi: 10.1086/159378

5. Bowen, G. H. 1988. The Mechanism of Mass Loss from Pulsating Cool Stars. In R. Stalio \& L. A. Willson (Eds.). Pulsation and mass loss in stars. 148:3. doi: 10.1007/978-94-009-3029-21

6. Kiss, C., Moor, A., \& Toth, L. V. 2004. VizieR Online Data Catalog: Far infrared loops in the 2nd Galactic Quadrant (Kiss+, 2004). VizieR Online Data Catalog, 1000.

7. Könyves,V.,Kiss,C.,Moór,A.,Kiss,Z.T.,\&Tóth,L.V. 2007. Catalogue of far-infrared loops in the Galaxy. Astronomy and Astrophysics.46: 1227-1234. doi: 10.1051/0004-6361:20065438

8. Wood, D. O. S., Myers, P. C., \& Daugherty, D. A.1994. IRAS images of nearby dark clouds. The Astrophysical Journal Supplement.95: 457-501.

9. Dupac, X., Bernard, J. P., Boudet, N., Giard, M., Lamarre, J. M., Meny, C., Pajot, F., Ristorcelli, I., Serra, G., Stepnik, B. \& Torre, J.

P. (2003). Inverse Temperature Dependence of the Dust Submillimeter Spectral Index. Astronomy \& Astrophysics. 404: 1115 .

10. Schnee, S. L., Ridge, N. A., Goodman, A. A. \& Jason, G. L.2005. A Complete Look at the Use of IRAS Emission Maps to Estimate Extinction and Dust Temperature. The Astrophysical Journal. 634: $442-450$.

11. Beichman, C. A.,Wilson, R. W.,Langer, W. D., \& Goldsmith,P. F.1983. Infrared limb brightening in the Barnard 5 cloud. The Astrophysical Journal Letters. 332: L81-L85.

12. Hildebrand, R. H. 1983. The determination of cloud mass and dust characteristics from sub millimeter thermal emission. Royal Astronomical Society. 24: 267-282.

13. Young, K., Phillips, T. G. \& Knapp, G.R.1993.Circumstellar Shells Resolved inIRAS Survey Data II. Analysis. Astrophysical Journal. 409: 725-738.

14. Aryal, B. \& Weinberger, R.2011.Dust structure around White

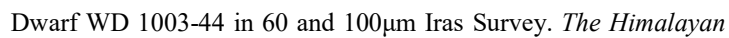
Physics. II: 5-10.

15. Gautam, A. K., \& Aryal, B.2019. A study of low-latitude $\left(|\ell|<10^{\circ}\right)$ far infrared cavities. Journal of Astrophysic $s$ and Astronomy. 40 (16):1-10. 